\title{
¿Cómo mejorar los resultados de pruebas objetivas en ingeniería? ¿Cómo mejorar el análisis y la relación entre conceptos de arquitectura? Dos ciclos de mejora docente
}

José AdOLFO HERRERA-MARTíN

Universidad de Sevilla

Departamento de Historia, Teoría y

Composición Arquitectónica

jaherrera@us.es

ORCID: https://orcid.org/0000-0003-0619-7011

D.O.I.: http://dx.doi.org/10.12795/JDU.2018.i01.70

Pp.: 1249-1271

\section{Resumen}

La presente comunicación describe dos ciclos de mejora, el primero desarrollado en la asignatura Valoraciones, Peritaciones y Tasaciones, correspondiente al cuarto curso del Grado en Edificación desarrollado en la Escuela Técnica Superior de Ingeniería de Edificación y el segundo desarrollado en la asignatura Historia, Teoría y Composiciones Arquitectónicas 2, correspondiente al segundo curso del Grado en Fundamentos de Arquitectura desarrollado en la Escuela Técnica Superior de Arquitectura de Sevilla. 
El primer ciclo de mejora pretende que los estudiantes identifiquen los conceptos que intervienen en la valoración del suelo, de las viviendas y de los locales, consulten, manejen y utilicen la normativa de aplicación, desarrollando cálculos y procedimientos para la obtención del valor de referencia de suelos, viviendas y locales.

El segundo ciclo de mejora se dirige a que los estudiantes, analicen, comparen y relacionen los diferentes espacios, usos y funciones en la vivienda, identificando tipos y estilos arquitectónicos.

Palabras Clave: Valoraciones, Peritaciones y Tasaciones, Historia, Teoría y Composiciones Arquitectónicas 2, Grado en Edificación, Grado en Fundamentos de Arquitectura, docencia universitaria, experimentación docente universitaria, habitar

\section{Contexto de la intervención}

El primer ciclo de mejora desarrollado en el curso 2018/2018, responde a la pregunta ¿cómo mejorar los resultados de las pruebas objetivas en el campo de la ingeniería?, continuando la innovación docente desarrollada en el curso 2017/2018, destinándolos ahora a la parte teórica de la asignatura, buscando mejorar el resultado de las pruebas objetivas establecidas en la programación docente.

Si bien los resultados de los CMD del curso 2017/2018 fueron satisfactorios observaba que los estudiantes tenían dificultades en las pruebas objetivas tipo test que forman parte del sistema de evaluación de la asignatura.

En el primer ciclo de mejora se han incorporado cambios en la metodología y en las actividades de los ciclos de mejora con objeto de alcanzar mejores resultados en las pruebas objetivas.

La innovación docente en la asignatura de Valoraciones, Tasaciones y Peritaciones comprendía la asignatura completa, pero por motivos de ajuste en la asignación del profesorado sólo se ha desarrollado una parte (22 horas). El segundo ciclo de mejora comprende también la asignatura 
completa de Historia, Teoría y Composiciones Arquitectónicas 2, habiéndose desarrollado el primer ciclo (12 horas).

El cómputo de las horas dirigidas a la innovación docente ha sido de $22+12$ horas $=34$ horas.

En la asignatura de Valoraciones, Tasaciones y Peritaciones, de cuarto curso, han participado un total de 67 (14 $+10+6+15+22$ ) estudiantes.

La asignatura de Valoraciones, Peritaciones y Tasaciones es el primer contacto que tienen los estudiantes con la cuantificación de valor del suelo, de la vivienda y los locales, y con la conformación económica de las certificaciones de obra y revisiones de precios (Herrera-Martín, 2017).

El aula cuenta con mobiliario formado por mesas de dibujo de baja altura y sillas sin anclar al piso del aula, que permite realizar cambios en la disposición del mobiliario. Cuenta con medios audiovisuales, cañón proyector, ordenador, conexión inalámbrica a internet y encerado (Herrera-Martín, 2017).

Los estudiantes cuentan con un libro publicado de la asignatura que contiene el desarrollo de las materias (Solis et al, 2013) y con material colgado en la plataforma de enseñanza virtual de la Universidad de Sevilla (Herrera-Martín, 2017).

Durante el curso 2017/2018 se realizó “modelo guía” (Herrera-Martín, 2017) que se entregó a los estudiantes en el aula y que está a disposición de los estudiantes en la plataforma de enseñanza virtual de la asignatura. Se obtuvieron buenos resultados en las pruebas prácticas de la asignatura.

En el curso 2017/2018 se realizó boletín preguntas tipo test que se facilitó a los estudiantes para mejorar los resultados de la parte teórica de la asignatura. El análisis del resultado de las pruebas tipo test arroja que hay casos de desajustes en las calificaciones de los estudiantes, observo estudiantes que presentan calificaciones muy buenas en la parte práctica de la asignatura y calificaciones muy bajas en la parte teórica de las pruebas tipo test. 
El diseño de los CMD de este curso 2018/2019 ha pretendido corregir la divergencia observada en el resultado de las calificaciones. Estableciendo cambios en la metodología y en el desarrollo de actividades.

La innovación docente se ha desarrollado en los días 25, 26 y 28 de septiembre, 2, 3, 5, 9, 10 y 16 de octubre.

En la asignatura de Historia, Teoría y Composiciones Arquitectónicas 2, de segundo curso, han participado 20 estudiantes.

La asignatura de Historia, Teoría y Composiciones Arquitectónicas 2, es una asignatura obligatoria de segundo curso del grado en Fundamentos de Arquitectura. El campo de estudio es la cuestión de habitar, desde el plano teórico de la arquitectura, desde la forma de habitar y desde propuestas arquitectónicas teniendo en cuenta consideraciones sociales, culturales, históricas, medioambientales y patrimoniales.

El aula cuenta con mobiliario formado por mesas de baja altura y sillas sin anclar al piso del aula, que permite realizar cambios en la disposición del mobiliario. Cuenta con cañón proyector, conexión inalámbrica a internet y encerado. Los alumnos pueden utilizar sus propios ordenadores o bien utilizar el préstamo de portátiles de la biblioteca.

La innovación docente se ha desarrollado en los días 25 de octubre, 8 y 15 de noviembre.

\section{Objetivos de los CMD}

Objetivos del ciclo de mejora de la asignatura de Valoraciones, Tasaciones y Peritaciones para los estudiantes:

- Identifiquen los conceptos que intervienen en la valoración del suelo.

- Relacionen los conceptos de valoraciones y la normativa de aplicación.

- Respondan de forma óptima a preguntas objetivas que relacionan conceptos de valoraciones en suelo. 
- Formulen múltiples preguntas a partir de la normativa y que sea de aplicación en la valoración de suelos.

- Formulen múltiples preguntas a partir de los resultados de la valoración de suelos y que se encuentre la base de la respuesta en la normativa de aplicación.

- Adquirieran manejo en el cálculo, en los procedimientos y en las operaciones necesarias para la obtención del valor de referencia de suelos.

- En la asignatura de Historia, Teoría y Composiciones Arquitectónicas 2:

- Identifiquen y relacionen los elementos y funciones de una vivienda.

- Analicen y propongan ejemplos de viviendas relevantes en la historia de la arquitectura.

- Expongan y realicen trabajos de análisis y comparación de modelos de viviendas.

- Participen en la propia evaluación del proceso de enseñanza aprendizaje.

\section{Principios didácticos}

En el proceso de innovación y mejora docente mantengo los principios didácticos del curso 2017/2018:

- Desde el punto de vista de los contenidos: deben estar centrados en la práctica y en el aprendizaje autónomo de los estudiantes; ser próximos en comprensión a los estudiantes; presentar una estructura clara y bien definida; y poder ser obtenidos a partir de la aplicación y realización de ejercicios prácticos en el aula, (HERRERA-MARTín, 2017).

- En relación con la metodología: se considera de gran importancia partir de preguntas y/o problemas que generen interés en los estudiantes; fomentar el debate para compartir conocimientos, aplicaciones y dudas en un clima de trabajo colaborativo; desarrollar el aprendizaje de los estudiantes mediante 
materiales docentes que sirvan de guía para la resolución de los problemas planteados, (HERRERA-MARTín, 2017).

- En la evaluación de los estudiantes: se considera relevante la participación e implicación en clase de los estudiantes en la resolución de los problemas planteados; la capacidad de síntesis y de anticipación en la resolución de los problemas propuestos, (HERRERA-MARTÍN, 2017).

CON OBJETO DE ALCANZAR LOS OBJETIVOS EXPRESADOS PARA EL CURSO 2018/2019, INCORPORO LOS SIGUIENTES PRINCIPIOS DIDÁCTICOS:

En el proceso de enseñanza-aprendizaje es necesario que los estudiantes adquieran la capacidad de formular preguntas que tengan como respuesta los contenidos objeto de aprendizaje. De manera que los contenidos queden fijados y conectados de forma sólida y estable en los estudiantes.

En el proceso de enseñanza-aprendizaje es necesario que los estudiantes participen en el proceso de su propia evaluación a fin de que conozcan y desarrollen los aspectos que deben reforzar y ejercitar en su aprendizaje.

\section{Mapa de contenidos y preguntas clave}

El mapa de contenidos y preguntas clave establecido para la asignatura de Valoraciones, Tasaciones y Peritaciones para este curso 2018/2019 se amplía respecto al curso pasado con las preguntas subrayadas en la tabla. Abordando el objetivo y el giro de planteamiento de la asignatura. El mapa de contenidos expresado en la figura 1 corresponde al primer ciclo de mejora programado de la asignatura de Valoraciones, Tasaciones y Peritaciones.

Jornadas de Formación e Innovación Docente del Profesorado | № 1 (2018)

(c) (i) (2) Esta obra se distribuye con la licencia Creative Commons Reconocimiento-NoComercial-SinObraDerivada $\quad 4.0$ Internacional (CC BY-NC-ND 4.0.) 
¿Qué es un Área de Reparto?

¿Qué es un Sector?

¿Qué relación existe entre áreas de reparto y sectores de una unidad de ejecución?

¿Qué es el aprovechamiento medio? ¿Cómo se obtiene?

¿Cuál es la superficie total de suelo de una unidad de ejecución?

¿Cuál es la superficie de suelo con aprovechamiento de una unidad de ejecución?

¿Cuál es la superficie de suelo público asociado a un área de reparto, y a un sector de una unidad de ejecución?

¿Qué es el aprovechamiento objetivo? ¿Cómo de obtiene?

¿Qué es el aprovechamiento subjetivo? ¿Cómo se obtiene?

¿Qué son usos lucrativos? ¿Cómo se obtienen? ¿Identifica los usos lucrativos de una unidad de ejecución?

¿Cuál es la edificabilidad total de una unidad de ejecución?

¿Qué es el coeficiente de ponderación? ¿Cómo se calcula?

¿Qué es el coeficiente de localización? ¿Cómo se calcula?

¿Cuál es el aprovechamiento que obtiene un propietario de suelo?

¿Cuántos metros cuadrados de uso lucrativo le corresponden de cada propietario de suelo por cada metro cuadrado de suelo?

¿Cuál es la edificabilidad que le corresponde a un propietario de suelo?

¿Cuál es la superficie de suelo correspondiente a las cesiones de un propietario de suelo?

¿Qué preguntas son necesarias para obtener el valor del suelo?

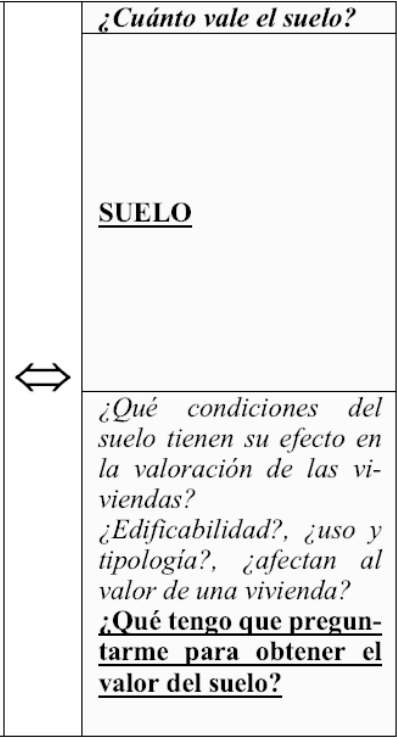

Figura 1: Mapa de contenidos y preguntas cuestionario (Fuente: Ampliación sobre la base Herrera-Martín, 2017)

El mapa de contenidos y preguntas clave establecido para la asignatura de Historia, Teoría y Composiciones Arquitectónicas 2 es el expresado en la figura 2.

\begin{tabular}{|l|l|l|}
\hline $\begin{array}{l}\text { ¿Qué relación se produce entre las partes de una vivienda?, ¿qué partes } \\
\text { puedo identificar?, ¿a qué corresponden cada una de sus partes? } \\
\text { ¿Uso igual a función? }\end{array}$ & ¿Cómo se habita? \\
$\begin{array}{l}\text { ¿Conoces algún "estilo arquitectónico" que se haya aplicado a la vi- } \\
\text { vienda? ¿puedes poner algunos ejemplos? }\end{array}$ \\
$\begin{array}{l}\text { ¿Encuentras alguna relación que se repita a lo largo de la historia en el el } \\
\text { diseño de la vivienda? }\end{array}$
\end{tabular} \mid \begin{tabular}{l} 
LA CASA \\
\hline ¿De qué se compone? \\
$\begin{array}{l}\text { iQué tengo que pregun- } \\
\text { tarme para tener una vi- } \\
\text { vienda? }\end{array}$
\end{tabular}

Figura 2: Mapa de contenidos y preguntas cuestionario. Historia, Teoría y Composiciones Ar-quitectónicas 2

\section{Modelo metodológico previo}

En cuanto a la impartición de la asignatura Valoraciones, Peritaciones y Tasaciones en el cur-so 2016-2017 fue eminentemente práctica y se fundamentó en la resolución de casos prácticos que se realizaron en clase con la 
ayuda de material docente elaborado por el profesor y con el apoyo del libro publicado de la asignatura.

En el curso 2018/2019 la docencia se dirigió hacia la parte teórica y hacia la parte práctica del bloque temático de valoración de suelo.

El desarrollo del ciclo de mejora quería resolver la dificultad de los estudiantes en la supera-ción de las pruebas objetivas tipo test (durante el curso 2017-2018 hubo estudiantes que no su-peraron la asignatura por la calificación obtenida en el apartado test).

La secuencia habitual del curso 2017/2018 se resume en la figura 2:

Pregunta global y planteamiento del problema + Identificación de aspectos esenciales del problema + Resolución de problemas con apoyo de modelo guía y explicaciones del profesor + Tiempo de trabajo de los estudiantes + Puesta en común y actividades de cierre

Figura 3. Secuencia de actividades de sesión docente habitual

El desarrollo del curso 2017/2018 siguió el siguiente esquema:

\begin{tabular}{|l|l|l|}
\hline \multicolumn{4}{|l|}{ Modelo de Sesión estándar } \\
\hline $\mathbf{1}$ & $\begin{array}{l}\text { Exposición del problema e identificación de los as- } \\
\text { pectos esenciales para su resolución }\end{array}$ & 20 minutos \\
\hline 2 & $\begin{array}{l}\text { Desarrollo de la resolución del problema con apoyo } \\
\text { de modelo guía y de explicaciones del profesor }\end{array}$ & 30 minutos \\
\hline 3 & $\begin{array}{l}\text { Puesta en común de las dificultades encontradas du- } \\
\text { rante la resolución del problema }\end{array}$ & 20 minutos \\
\hline 4 & $\begin{array}{l}\text { Desarrollo por los estudiantes de caso práctico ayu- } \\
\text { dándose de modelo guía, anotaciones y explicaciones } \\
\text { del profesor }\end{array}$ & 30 minutos \\
\hline 5 & $\begin{array}{l}\text { Actividades de cierre, puesta en común de las dificul- } \\
\text { tades encontradas, de los procedimientos y de los re- } \\
\text { sultados del caso práctico }\end{array}$ & 20 minutos \\
\hline
\end{tabular}

Figura 4. Estructura de sesión estándar (Herrera-Martín, 2017)

El desarrollo de los CMD del curso 2017/2018 me llevó a las siguientes consideraciones: 
- El "modelo guía de trabajo" en papel se ha observado de gran relevancia para el me-jor y mayor entendimiento y comprensión por los estudiantes (Herrera-Martín, 2017).

- El modelo guía ha propiciado que los estudiantes se centren en la tarea encomendada, consiguiendo un mayor aprovechamiento de la enseñanza docente (Herrera-Martín, 2017).

- Observo necesario aumentar el tiempo asignado al desarrollo de trabajo en el aula por parte de los estudiantes en la resolución de los problemas (Herrera-Martín, 2017).

- Observo necesario ampliar los contenidos del modelo guía para hacer frente a posibles saltos en los niveles iniciales de aprendizajes de los estudiantes (Herrera-Martín, 2017).

En cuanto a la impartición de la asignatura de Historia, Teoría y Composición Arquitectónicas 2, no he tenido con anterioridad docencia, partiendo por tanto de modelos previos similares a los expresados para la asignatura de Valoraciones, Tasaciones y Peritaciones.

\section{Modelo metodológico habitual}

El modelo metodológico desarrollado en la asignatura de Valoraciones, Tasaciones y Perita-ciones se resume en la siguiente tabla

\begin{tabular}{|l|l|}
\hline \multicolumn{2}{|l|}{ Estructura de los ciclos de mejora } \\
\hline Actividades de iniciación & $\begin{array}{l}\text { Introducción, exposición de teoría y de con- } \\
\text { ceptos prácticos necesarios para responder a } \\
\text { las preguntas tipo test y para resolver los ejer- } \\
\text { cicios de problemas }\end{array}$ \\
\hline $\begin{array}{l}\text { Actividades de } \\
\text { profundización }\end{array}$ & $\begin{array}{l}\text { Desarrollo de acciones, reflexiones y procedi- } \\
\text { mientos para analizar el contenido teórico de } \\
\text { la asignatura y para su aplicación en ejerci- } \\
\text { cios prácticos de la asignatura }\end{array}$ \\
\hline
\end{tabular}

Jornadas de Formación e Innovación Docente del Profesorado | № 1 (2018) Esta obra se distribuye con la licencia Creative Commons 


\begin{tabular}{|l|l|}
\hline $\begin{array}{l}\text { Actividades de } \\
\text { seguimiento }\end{array}$ & $\begin{array}{l}\text { Realización de problemas y respuesta a } \\
\text { preguntas con ayuda de modelo guía y } \\
\text { bibliografía }\end{array}$ \\
\hline $\begin{array}{l}\text { Actividades de identifica- } \\
\text { ción de dificultades y de } \\
\text { refuerzo }\end{array}$ & $\begin{array}{l}\text { Identificación de las dificultades comunes de } \\
\text { los estudiantes a la hora de resolver el pro- } \\
\text { blema o preguntas propuestas. Exposición } \\
\text { de procedimientos para resolver problemas y } \\
\text { aclarar preguntas }\end{array}$ \\
\hline Actividades de cierre & $\begin{array}{l}\text { Resumen de aspectos, contenidos y procedi- } \\
\text { mientos básicos para el desarrollo de la prác- } \\
\text { tica correspondiente a la sesión desarrollada }\end{array}$ \\
\hline
\end{tabular}

Figura 5. Estructura de actividades del modelo metodológico habitual

\section{Modelo metodológico ideal}

El modelo metodológico ideal de la asignatura de Valoraciones, Tasaciones y Peritaciones se dirige hacia la consecución de los objetivos marcados para el desarrollo de la secuencia de los ciclos de innovación docente.

El modelo ideal continúa por un lado con las premisas establecidas para la parte práctica de la asignatura desarrolladas en el curso 2017/2018:

- Desarrollo de "secuencia de preguntas" destinadas a identificar los elementos princi-pales a resolver del problema del caso práctico. Aspectos que van desde la aplicación de la normativa hasta la identificación de la relación entre los elementos necesarios pa-ra resolverlo (Herrera-Martín, 2017).

- Asignación de tiempo a los estudiantes para que identifiquen los datos necesarios para resolver el problema (Herrera-Martín, 2017).

- Exposición y análisis sobre "modelo guía" que se entrega a los estudiantes al inicio de la clase sobre el procedimiento para resolver el problema planteado. Los estudiantes siguen la resolución del caso práctico ayudándose de las explicaciones del profesor y del modelo guía que se les entrega (Herrera-Martín, 2017). 
- Asignación de tiempo destinado a que los estudiantes resuelvan otro caso similar ayu-dándose del modelo guía entregado y de las aportaciones del profesor (Herrera-Martín, 2017).

- Puesta en común de los resultados, debate sobre las dificultades obtenidas y resolución del caso mediante debate y puesta en común. Los errores que se hayan advertido du-rante la resolución del problema se entienden como una oportunidad de mejora en el proceso de enseñanza-aprendizaje (Herrera-Martín, 2017).

Y por otro lado incorpora nuevos valores a la parte teórica de la asignatura:

- Identificación y exposición de los conceptos básicos necesarios para la valoración de un suelo, de una vivienda y de un local.

- Exposición de preguntas destinadas a relacionar conceptos teóricos y su regulación en textos legales y normativos.

- Asignación de tempo destinado a que los estudiantes analicen los conceptos teóricos, los textos legales y normativos, realizando esquemas y mapas de contenidos.

- Aplicación práctica de los conceptos teóricos, mediante la realización de batería de pre-guntas de relación entre contenidos.

- Elaboración de guía de preguntas por los estudiantes para los conceptos teóricos expues-tos.

El modelo metodológico ideal para la asignatura de Historia, Teoría y Composiciones Arquitectónicas 2, se resume en la figura 6.

Presentación del trabajo a realizar + exposición de medios y ámbito de estudio + profundización en análisis y comparación entre elementos + síntesis del trabajo realizado + evaluación propia del proceso de aprendizaje

Figura 6. Secuencia metodológica ideal en la asignatura de Historia, Teoría y Composiciones Arquitectónicas 2

Jornadas de Formación e Innovación Docente del Profesorado | № 1 (2018) Esta obra se distribuye con la licencia Creative Commons 


\section{Modelo metodológico posible}

El modelo metodológico posible tiene en cuenta las limitaciones de tiempo y la asignación de horas de trabajo de los estudiantes, buscando mejorar el modelo habitual acercándose al modelo ideal (Herrera-Martín, 2017).

El modelo metodológico para la asignatura de Valoraciones, Tasaciones y Peritaciones com-bina por un lado la experiencia del curso 2017/2018 en la aplicación práctica de la asignatura y ha incorporado en el curso 2018/2019 la profundización en la parte teórica, consignando tiempo destinado a la respuesta de preguntas relacionadas con conceptos teóricos y también a la formu-lación de preguntas sobre conceptos teóricos.

Se busca en la parte teórica que, en el tiempo asignado en clase, los estudiantes tomen el rol de la persona que elabora el test de evaluación, buscando todas las preguntas y respuestas posi-bles relacionadas con los conceptos teóricos contemplados en la asignatura. Desarrollando bate-ría de preguntas-respuestas vinculadas a los conceptos teóricos expuestos durante la sesión.

Se plantea la siguiente "distribución tipo de actividades" dentro del modelo metodológico posible (figura 7):

\begin{tabular}{|l|l|l|}
\hline \multicolumn{3}{|l|}{ Modelo de Sesión estándar } \\
\hline 1 & $\begin{array}{l}\text { Exposición de conceptos teóricos e identificación de } \\
\text { aspectos esenciales. Exposición apoyada en la formu- } \\
\text { lación de preguntas }\end{array}$ & 30 minutos \\
\hline 2 & $\begin{array}{l}\text { Resolución de problemas con apoyo de modelo guía, } \\
\text { normativa y textos legales y explicaciones del profesor }\end{array}$ & 20 minutos \\
\hline 3 & $\begin{array}{l}\text { Desarrollo por los estudiantes de caso práctico ayu- } \\
\text { dándose de modelo guía, anotaciones y explicaciones } \\
\text { del profesor }\end{array}$ & 20 minutos \\
\hline 4 & $\begin{array}{l}\text { Realización de mapa de contenidos y conceptos teóri- } \\
\text { cos por los estudiantes. Identificación de dificultades. } \\
\text { Puesta en común de resultados y criterios aplicados }\end{array}$ & 20 minutos \\
\hline
\end{tabular}

Jornadas de Formación e Innovación Docente del Profesorado | № 1 (2018) Esta obra se distribuye con la licencia Creative Commons Reconocimiento-NoComercial-SinObraDerivada Internacional (CC BY-NC-ND 4.0.) 


\begin{tabular}{|l|l|l|}
\hline 5 & Actividades de consolidación y cierre. & 30 minutos \\
& Elaboración de preguntas dirigidas hacia los concep- & \\
tos expresados en el mapa de contenidos de la sesión. & \\
& Elaboración de posibles respuestas a las preguntas & \\
realizadas. & & \\
Realización de preguntas y respuestas por los estu- & \\
diantes relacionadas con el contenido de la sesión. & \\
Realización de simulacro tipo test incluido en el mo- & \\
delo guía. &
\end{tabular}

Figura 7. Estructura de sesión estándar

\section{Secuencia de actividades de los ciclos de mejora}

La estructura de la asignatura completa de Valoraciones, Tasaciones y Peritaciones sigue la siguiente estructura de ciclos encadenados:

\begin{tabular}{|c|c|c|}
\hline \multicolumn{3}{|c|}{ Estructura de los ciclos de mejora } \\
\hline Primer ciclo de mejora & ¿Cuánto vale el suelo? & $\begin{array}{l}\text { Sesión 1a ( } 2 \text { horas) } \\
\text { Sesión 2a ( } 2 \text { horas) } \\
\text { Sesión 3a ( } 2 \text { horas) }\end{array}$ \\
\hline $\begin{array}{l}\text { Segundo ciclo de mejora } \\
\text { (Primera parte) }\end{array}$ & $\begin{array}{l}\text { ¿Cuánto vale una } \\
\text { vivienda? }\end{array}$ & $\begin{array}{l}\text { Sesión 4á ( } 2 \text { horas) } \\
\text { Sesión } 5 a \text { a ( } 2 \text { horas) } \\
\text { Sesión 6a (2horas) }\end{array}$ \\
\hline $\begin{array}{l}\text { Segundo ciclo de mejora } \\
\text { (Segunda parte) }\end{array}$ & ¿Cuánto vale un local? & $\begin{array}{l}\text { Sesión 7ạ ( } 2 \text { horas) } \\
\text { Sesión 8a ( } 2 \text { horas) }\end{array}$ \\
\hline DURACIÓN TOTAL & & 16 HORAS \\
\hline
\end{tabular}

Figura 8. Estructura de los ciclos de mejora de la asignatura de Valoraciones, Tasaciones y Peri-taciones.

Se establecen dos ciclos de mejora, un primer ciclo de mejora compuesto por cuatro sesiones de dos horas dirigidos a obtener el valor del suelo y un segundo ciclo de mejora compuesto por dos partes, la primera parte dirigida hacia el valor de una vivienda y la segunda parte dirigida hacia el valor de un local. Por motivos de ajuste de profesorado se ha realizado el primer ciclo de mejora.

El resto de horas de innovación docente se han realizado en la asignatura de Historia, Teoría y Composiciones 
Arquitectónicas 2. En la figura 8 se tiene la estructura completa de ciclos de mejora en la asignatura (figura 9).

\begin{tabular}{|c|c|c|}
\hline \multicolumn{3}{|c|}{ Estructura de los ciclos de mejora } \\
\hline Primer ciclo de mejora & ¿Qué es una vivienda? & $\begin{array}{l}\text { Sesión 1a ( } 4 \text { horas) } \\
\text { Sesión 2a ( } 4 \text { horas) } \\
\text { Sesión 3ạ (4 horas) }\end{array}$ \\
\hline $\begin{array}{l}\text { Segundo ciclo de } \\
\text { mejora }\end{array}$ & $\begin{array}{l}\text { ¿Qué relaciones se produ- } \\
\text { cen entre viviendas? }\end{array}$ & $\begin{array}{l}\text { Sesión 4a ( } 4 \text { horas) } \\
\text { Sesión } 5 \underline{a} \text { ( } 4 \text { horas) } \\
\text { Sesión 6ạ (4horas) }\end{array}$ \\
\hline Tercer ciclo de mejora & ¿Cuánto vale un local? & $\begin{array}{l}\text { Sesión 7a ( } 4 \text { horas) } \\
\text { Sesión } 8 \text { a ( } 4 \text { horas) } \\
\text { Sesión } 9 \underline{a} \text { ( } 4 \text { horas })\end{array}$ \\
\hline DURACIÓN TOTAL & & 36 HORAS \\
\hline
\end{tabular}

Figura 9. Estructura de los ciclos de mejora de la asignatura de Historia, Teoría y Composicio-nes Arquitectónicas 2.

\section{Cuestionario inicial y final}

Con objeto de valorar el proceso de los CMD se desarrollan los siguientes cuestionarios:

Cuestionario del ciclo de mejora de la asignatura Valoraciones, Tasaciones y Peritaciones:

1. ¿Qué es un Área de Reparto?
2. ¿Qué es un Sector?
3. ¿Qué relación existe entre áreas de reparto y sectores de una unidad de
ejecución?
4. ¿Qué es el aprovechamiento medio? ¿Cómo se obtiene?
5. ¿Cuál es la superficie total de suelo de una unidad de ejecución?
6. ¿Cuál es la superficie de suelo con aprovechamiento de una unidad de
ejecución?
7. ¿Cuál es la superficie de suelo público asociado a un área de reparto, y a
un sector de una unidad de ejecución?
8. ¿Qué es el aprovechamiento objetivo? ¿Cómo de obtiene?
9. ¿Qué es el aprovechamiento subjetivo? ¿Cómo se obtiene?

Jornadas de Formación e Innovación Docente del Profesorado | № 1 (2018) Esta obra se distribuye con la licencia Creative Commons Reconocimiento-NoComercial-SinObraDerivada Internacional (CC BY-NC-ND 4.0.) 
10. ¿Qué son usos lucrativos? ¿Cómo se obtienen? ¿ldentifica los usos lucrativos de una unidad de ejecu-ción?

11. ¿Cuál es la edificabilidad total de una unidad de ejecución?

12. ¿Qué es el coeficiente de ponderación? ¿Cómo se calcula?

13. ¿Qué es el coeficiente de localización? ¿Cómo se calcula?

14. ¿Cuál es el aprovechamiento que obtiene un propietario de suelo?

15. ¿Cuántos metros cuadrados de uso lucrativo le corresponden de cada propietario de suelo por cada metro cuadrado de suelo?

16. ¿Cuál es la edificabilidad que le corresponde a un propietario de suelo?

17. ¿Cuál es la superficie de suelo correspondiente a las cesiones de un propietario de suelo?

18. ¿Qué preguntas son necesarias para obtener el valor del suelo?

Figura 10. Cuestionario del ciclo de mejora de la asignatura de Valoraciones, Tasaciones y Peri-taciones

Cuestionario del ciclo de mejora de la asignatura de Historia, Teoría y Composiciones Arquitec-tónicas 2:

1. ¿Qué relación se produce entre las partes de una vivienda?, ¿qué partes puedo identificar?, ¿a qué corres-ponden cada una de sus partes?

2. ¿Uso igual a función?

3. ¿Conoces algún "estilo arquitectónico" que se haya aplicado a la vivienda?, ¿puedes poner algunos ejem-plos?

4. ¿Encuentras alguna relación que se repita a lo largo de la historia en el diseño de la vivienda?

Figura 11. Cuestionario del ciclo de mejora de la asignatura de Historia, Teoría y Composicio-nes Arquitectónicas 2.

\section{Actividades de los CMD}

En este apartado se desarrollan, la primera sesión del ciclo de mejora de la asignatura de Va-loraciones, Tasaciones y Peritaciones y la última sesión del ciclo de mejora de la asignatura de Historia, Teoría y Composiciones Arquitectónicas 2.

En la siguiente tabla se incluye: la pregunta del cuestionario de diagnóstico del nivel inicial de los estudiantes 
(C); las tareas que el estudiante debía realizar (T); las acciones a realizar por el docente $(A)$ y actividades de cierre (CR).

La primera sesión del ciclo de mejora quedó enmarcada con la pregunta “¿Cuánto vale el suelo?"

\begin{tabular}{|c|c|c|}
\hline & & ¿CUÁNTO VALE EL SUELO? \\
\hline & & Sesión 1ạ \\
\hline & dad & $\begin{array}{l}\text { Preguntas }(C) \text {, tareas para los estudiantes }(T) \text {, acciones docentes } \\
\text { (A) y de cierre (CR) }\end{array}$ \\
\hline 1 & & $\begin{array}{l}\text { Realización de cuestionario inicial } \\
\text { (20 minutos) }\end{array}$ \\
\hline 2 & C & $\begin{array}{l}\text { ¿Qué es un Área de Reparto? } \\
\text { ¿Qué es un Sector? } \\
\text { ¿Qué relación existe entre áreas de reparto y sectores de una } \\
\text { unidad de ejecución? }\end{array}$ \\
\hline & $\mathrm{T}$ & $\begin{array}{l}\text { 1. Identificar en el modelo guía, a partir de las explicaciones del } \\
\text { profesor, las relaciones entre los conceptos de área de reparto, } \\
\text { sector y unidad de ejecución } \\
\text { 2. Localizar los datos y elementos que distinguen cada área de } \\
\text { reparto, sector y unidad de ejecución en el modelo guía } \\
\text { 3. Identificación sobre el modelo "mudo" de los elementos que } \\
\text { identifican al área de reparto, sector y unidad de ejecución } \\
\text { 4. Realización de esquemas que identifiquen las relaciones entre } \\
\text { los conceptos de área de reparto, sector y unidad de ejecución } \\
\text { 5. Señalar las áreas, sectores y unidades de ejecución que inter- } \\
\text { vienen en el problema mudo. } \\
\text { 6. Exposición de preguntas tipo test relacionadas con los con- } \\
\text { ceptos expuestos } \\
\text { (20 minutos) }\end{array}$ \\
\hline
\end{tabular}

Jornadas de Formación e Innovación Docente del Profesorado | № 1 (2018) Esta obra se distribuye con la licencia Creative Commons Reconocimiento-NoComercial-SinObraDerivada Internacional (CC BY-NC-ND 4.0.) 


\begin{tabular}{|c|}
\hline A \\
1. Proyección de diapositiva con imagen de modelo guía \\
2. Identificación sobre el modelo guía de los conceptos de área \\
de reparto, sector y unidad de ejecución \\
3. Descripción de las relaciones que se producen entre áreas de \\
reparto, sectores y unidades de ejecución \\
4. Realización de esquemas y diagramas sintéticos en los que se \\
relacionan los conceptos trabajados. Indicación de los elemen- \\
tos principales \\
5. Identificación de los elementos que identifican a un área de \\
reparto, a un sectory a una unidad de ejecución \\
6. Seguimiento y tutela de la resolución del modelo mudo por \\
los estudiantes \\
7. Formulación de preguntas sobre la base de conceptos \\
indicados \\
(20 minutos) \\
¿Qué es el aprovechamiento medio? ¿Cómo se obtiene? \\
¿Cuál es la superficie total de suelo de una unidad de ejecución? \\
¿Cuál es la superficie de suelo con aprovechamiento de una uni- \\
dad de ejecución? \\
¿Cuál es la superficie de suelo público asociado a un área de re- \\
parto, y a un sector de una unidad de ejecución?
\end{tabular}

Jornadas de Formación e Innovación Docente del Profesorado | № 1 (2018) Esta obra se distribuye con la licencia Creative Commons Reconocimiento-NoComercial-SinObraDerivada 


\begin{tabular}{|c|c|c|}
\hline & A & $\begin{array}{l}\text { 1. Proyección de diapositiva de imagen de modelo guía } \\
\text { 2. Descripción sobre modelo guía de los conceptos de aprove- } \\
\text { chamiento y uso lucrativo. Identificación de las relaciones en- } \\
\text { tre conceptos. } \\
\text { 3. Descripción de procedimientos para la obtención de aprove- } \\
\text { chamientos urbanísticos y descripción de superficies asignadas } \\
\text { 4. Descripción de las relaciones entre las partes. Desarrollo de } \\
\text { un esquema } \\
\text { 5. Realización del esquema en la pizarra con indicación de los } \\
\text { elementos principales a tener en cuenta en la resolución del } \\
\text { problema de aprovechamientos } \\
\text { 6. Resolución de problema de aprovechamiento sobre modelo } \\
\text { guía. Identificación de errores advertidos y soluciones } \\
\text { 7. Formulación de preguntas sobre la base de conceptos } \\
\text { indicados } \\
\text { (20 minutos) }\end{array}$ \\
\hline \multirow[t]{2}{*}{4} & CR & Actividades de cierre \\
\hline & $\mathrm{T}$ & $\begin{array}{l}\text { 1. Puesta en común de las dificultades encontradas } \\
\text { 2. Resumen de preguntas y respuestas dadas a los conceptos } \\
\text { expuestos } \\
\text { (20 minutos) }\end{array}$ \\
\hline & A & $\begin{array}{l}\text { 1. Realización de simulacro tipo test de los conceptos expues- } \\
\text { tos. Solucionario del simulacro tipo test. } \\
\text { ( } 20 \text { minutos) }\end{array}$ \\
\hline
\end{tabular}

Figura 12. Secuencia de actividades del ciclo de mejora de la asignatura de Valoraciones, Tasa-ciones y Peritaciones

Las actividades del ciclo de mejora de la asignatura de Historia, Teoría y Composiciones Arquitectónicas 2 se describen en la figura 13

\begin{tabular}{|l|l|}
\hline \multicolumn{2}{|c|}{ ¿CÓMO SE HABITA? } \\
\hline Sesión 3a
\end{tabular}

Jornadas de Formación e Innovación Docente del Profesorado | № 1 (2018) Esta obra se distribuye con la licencia Creative Commons Reconocimiento-NoComercial-SinObraDerivada Internacional (CC BY-NC-ND 4.0.) 


\begin{tabular}{|c|c|c|}
\hline & & $\begin{array}{l}\text { 3. Realización de trabajo de análisis de tres viviendas } \\
\text { por equipos para su posterior exposición en clase } \\
\text { (100 minutos) }\end{array}$ \\
\hline & $\mathrm{T}$ & $\begin{array}{l}\text { 1. Exposición de los trabajos realizados por los } \\
\text { estudiantes. } \\
\text { 2. Evaluación de los trabajos expuestos por los estu- } \\
\text { diantes. Los estudiantes evalúan el trabajo realizado } \\
\text { por los compañeros, siendo su evaluación parte de la } \\
\text { calificación de los trabajos. } \\
\text { 3. El profesor profundiza sobre la exposición de los tra- } \\
\text { bajos y evalúa la exposición de los trabajos. }\end{array}$ \\
\hline & & $\begin{array}{l}\text { 4. Durante la exposición se realiza debate sobre la expo- } \\
\text { sición realizada y se resuelven dudas. } \\
\text { (100 minutos) }\end{array}$ \\
\hline \multirow[t]{2}{*}{3} & $C$ & $\begin{array}{l}\text { ¿Qué relación se produce entre las partes de una vi- } \\
\text { vienda?, ¿qué partes puedo identificar?, ¿a qué corres- } \\
\text { ponden cada una de sus partes? } \\
\text { ¿Uso igual a función? } \\
\text { ¿Conoces algún "estilo arquitectónico" que se haya apli- } \\
\text { cado a la vivienda?, ¿puedes poner algunos ejemplos? } \\
\text { ¿Encuentras alguna relación que se repita a lo largo de } \\
\text { la historia en el diseño de la vivienda? }\end{array}$ \\
\hline & $\mathrm{T}$ & $\begin{array}{l}\text { 1. Los estudiantes responden a las preguntas del cues- } \\
\text { tionario, previamente facilitado. } \\
\text { ( } 40 \text { minutos) }\end{array}$ \\
\hline
\end{tabular}

Figura 13. Secuencia de actividades del ciclo de mejora de la asignatura de Historia, Teoría Y Composiciones Arquitectónicas 2.

\section{Seguimiento de la evolución de los modelos mentales de los estudiantes, de su proceso de aprendizaje a los largo de los CMD}

Para la valoración de la evolución del proceso de enseñanza-aprendizaje de los estudiantes se plantearon los siguientes niveles de aprendizaje.

Para la asignatura de Valoraciones, Tasaciones y Peritaciones, el esquema marcado en la figura 14.

\begin{tabular}{|l|l|}
\hline Niveles de la Escalera de Aprendizaje \\
\hline Niveles & Corresponde a estudiantes que: \\
\hline
\end{tabular}

Jornadas de Formación e Innovación Docente del Profesorado | № 1 (2018) Esta obra se distribuye con la licencia Creative Commons Reconocimiento-NoComercial-SinObraDerivada $\quad 4.0$ Internacional (CC BY-NC-ND 4.0.) 


\begin{tabular}{|l|l|}
\hline Nivel 1 & $\begin{array}{l}\text { No poseen ningún conocimiento sobre la materia } \\
\text { Responden en blanco o sin sentido }\end{array}$ \\
\hline Nivel 2 & Poseen un conocimiento inicial de carácter general e intuitivo \\
\hline Nivel 3 & $\begin{array}{l}\text { Poseen un conocimiento concreto y bien dirigido sobre la } \\
\text { materia }\end{array}$ \\
\hline Nivel 4 & $\begin{array}{l}\text { Amplían el conocimiento sobre parte de la materia mediante } \\
\text { aportaciones individuales en una parte de la misma }\end{array}$ \\
\hline Nivel 5 & $\begin{array}{l}\text { Destacan de forma excepcional por su conocimiento y aplica- } \\
\text { ción de la materia, producto de su trabajo y reflexión sobre el } \\
\text { objeto de estudio }\end{array}$ \\
\hline
\end{tabular}

Figura 14. Niveles de la escalera de aprendizaje (Herrera-Martín, 2017)

Para la asignatura de Historia, Teoría y Composiciones Arquitectónicas 2, el esquema mar-cado en la figura 15

\begin{tabular}{|l|c|c|c|c|}
\hline & \multicolumn{4}{|c|}{ Número (porcentaje) de estudiantes } \\
\hline NIVEL 4 & $3(15 \%)$ & $2(10 \%)$ & $7(35 \%)$ & $0(0 \%)$ \\
\hline NIVEL 3 & $2(10 \%)$ & $1(5 \%)$ & $7(35 \%)$ & $1(5 \%)$ \\
\hline NIVEL 2 & $15(75 \%)$ & $10(50 \%)$ & $5(25 \%)$ & $11(55 \%)$ \\
\hline NIVEL 1 & $0(0 \%)$ & $7(35 \%)$ & $1(5 \%)$ & $8(40 \%)$ \\
\hline & PREGUNTA 1 & PREGUNTA 2 & PREGUNTA 3 & PREGUNTA 4 \\
\hline
\end{tabular}

Figura 15. Niveles de la escalera de aprendizaje

\section{Evaluación del propio diseño y de mi intervención}

Aunque el desarrollo de los ciclos de mejora se ha visto afectado por cambios en la asigna-ción del profesorado, los resultados de la innovación docente han permitido verificar mejoras en los resultados respecto pasado en la asignatura de Valoraciones, Tasaciones y Peritaciones. En la al curso asignatura de Historia, Teoría y Composición Arquitectónicas 2, el resultado de la invocación ha sido muy favorable, habiendo superado el primer bloque de la asignatura el 100\% de los estudiantes. 
El resultado de los cuestionarios queda resumido en la figura 16.

\begin{tabular}{|l|c|c|c|c|}
\hline & \multicolumn{4}{|c|}{ Número (porcentaje) de estudiantes } \\
\hline NIVEL 4 & $3(15 \%)$ & $2(10 \%)$ & $7(35 \%)$ & $0(0 \%)$ \\
\hline NIVEL 3 & $2(10 \%)$ & $1(5 \%)$ & $7(35 \%)$ & $1(5 \%)$ \\
\hline NIVEL 2 & $15(75 \%)$ & $10(50 \%)$ & $5(25 \%)$ & $11(55 \%)$ \\
\hline NIVEL 1 & $0(0 \%)$ & $7(35 \%)$ & $1(5 \%)$ & $8(40 \%)$ \\
\hline & PREGUNTA 1 & PREGUNTA 2 & PREGUNTA 3 & PREGUNTA 4 \\
\hline
\end{tabular}

Figura 16. Resultado cuestionario final bloque I, asignatura Historia, Teoría y Composiciones Arquitectónicas 2.

Realizada la innovación docente el $60 \%$ de los estudiantes se sitúa por encima del nivel 1, estando el $70 \%$ de los estudiantes por encima del nivel 2 en la pregunta 3.

El resultado de los cuestionarios difiere respecto al resultado del trabajo realizado por equi-pos donde el resultado obtenido es mucho mejor.

La valoración conjunta de resultados queda expresada en la figura 17.

\begin{tabular}{|l|c|c|}
\hline & Número de estudiantes & \% de estudiantes \\
\hline NIVEL (9-10) & 0 & 0 \\
\hline NIVEL (8-9) & 8 & 40 \\
\hline NIVEL (7-8) & 11 & 55 \\
\hline NIVEL (5-7) & 1 & 5 \\
\hline NIVEL (0-5) & 0 & 0 \\
\hline & & \\
\hline
\end{tabular}

Figura 17. Resultado de calificaciones de bloque I, asignatura Historia, Teoría y Composiciones Arquitectónicas 2.

El 95\% de los estudiantes se sitúa en una calificación entre 7 y 9 sobre 10 .

El resultado obtenido, aun siendo favorable permite identificar las siguientes líneas de mejora: 
- Establecer mayor tiempo en clase al debate teórico y argumentado sobre los modos de habitar en la historia apoyado sobre referencias, tipologías y estilos arquitectónicos.

- Establecer dentro de la parte práctica espacio destinado a la realización de ensayo crítico sobre el tratamiento de la vivienda en la historia de la arquitectura.

\section{Conclusiones finales. Aspectos de la experiencia se pretenden incorporar a la práctica docente habitual.}

Respecto a la innovación docente llevada a cabo en la asignatura de Valoraciones, Tasacio-nes y Peritaciones, se observa una mejoría respecto a la situación del curso pasado 2017-2018, pero no ha sido posible contrastar los resultados obtenidos debido a cambios en la asignación del profesorado durante la realización del ciclo de mejora.

Respecto a la innovación docente llevada a cabo en la asignatura de Historia, Teoría y Com-posiciones Arquitectónicas 2 se observa muy adecuado que los propios estudiantes participen y profundicen en su propio aprendizaje y que también formen parte del proceso de evaluación.

Los estudiantes al trabajar para superar la asignatura y adquirir los conocimientos, compe-tencias y capacidades son plenamente aptos para identificar las dificultades del propio aprendiza-je y de observar el nivel de sus semejantes en el proceso de enseñanza-aprendizaje.

Considero una aportación que pretendo incorporar a la práctica docente habitual que los es-tudiantes formen parte del proceso de evaluación y superación de la asignatura.

Jornadas de Formación e Innovación Docente del Profesorado I № 1 (2018)

Esta obra se distribuye con la licencia Creative Commons Reconocimiento-NoComercial-SinObraDerivada Internacional (CC BY-NC-ND 4.0.) 


\section{Bibliografía}

Bain, K. (2004). What the Best Collage Teachers Do?. Cambridge, MA: Harvard University Press. (Trad. cast.: Lo que hacen los mejores profesores universitarios. Valencia: Publica-ciones de la Universidad de Valencia, 2005).

Finkel, D. (2000). Teaching with Your Mouth Shut. Portsmouth, NH: Heinemann Boynton/Cook. (Trad. cast.: Dar clase con la boca cerrada. Valencia: Publicaciones de la Universidad de Valencia, 2008)

Herrera-Martín, J. A. (2016). ¿Cómo implantamos las instalaciones en el edificio? Representa-ción gráfica, trazado y dimensiones. En R. Porlán y E. Navarro (Coords.), III Jornadas de Docencia Universitaria (págs. 730-745). Sevilla: Instituto de Ciencias de la Educación de la Universidad de Sevilla.

Herrera-Martín, J. A. (2017). ¿Cuánto vale el suelo, una vivienda o un local? En R. Porlán y E. Navarro (Coords.), IV Jornadas de Docencia Universitaria (págs. 937-956). Sevilla: Institu-to de Ciencias de la Educación de la Universidad de Sevilla.

Solís, J.A. et al. (2013). Las valoraciones inmobiliarias. Suelo y edificación. Sevilla: Fundación Cultural del Colegio Oficial de Aparejadores y Arquitectos Técnicos de Sevilla.

Jornadas de Formación e Innovación Docente del Profesorado | № 1 (2018) Esta obra se distribuye con la licencia Creative Commons 